\title{
Lactobacillus vaginalis sp. nov. from the Human Vagina
}

\author{
T. MARTIN EMBLEY, ${ }^{1 *}$ NAZIR FAQUIR, ${ }^{1}$ WALTER BOSSART ${ }^{2}$ AND MATTHEW D. COLLINS ${ }^{3}$ \\ Microbial Technology Research Unit, North East London Polytechnic, Stratford, London E15 4LZ, ${ }^{1}$ and Division of \\ Microbiology, AFRC Institute of Food Research, Reading Laboratory, Shinfield, Reading RG2 9AT, ${ }^{3}$ United Kingdom, \\ and Vaccine Division, Solco Basle Ltd., CH 4127 Birsfelden, Switzerland ${ }^{2}$
}

\begin{abstract}
Biochemical, chemical, and nucleic acid analyses were carried out on some lactobacilli isolated from the vaginas of patients suffering from trichomoniasis. Phenotypically the strains resembled Lactobacillus fermentum and $L$. reuteri. Nucleic acid studies, however, showed that the vaginal strains were distinct from these and all other lactobacilli examined and therefore warrant a new species. The name Lactobacillus vaginalis sp. nov. is proposed. The type strain of $L$. vaginalis is NCTC 12197.
\end{abstract}

In the course of investigations aimed at developing a vaccine (Solco Trichovac/Gynatren; Solco Basle Ltd., Birsfelden, Switzerland) against recurrent vaginitis, a number of lactobacilli were isolated from vaginal swabs from patients with trichomoniasis. These strains showed similarities with Lactobacillus fermentum, a species commonly isolated from the vagina. L. fermentum, however, exhibits considerable phenotypic diversity (6), and the assignment of the vaginal strains to this species is uncertain. In view of this, we investigated the relationship of the vaginal strains to representatives of L. fermentum and other heterofermentative lactobacilli by using biochemical, chemical, and nucleic acid techniques. The results indicated that the vaginal strains form a new species, for which the name Lactobacillus vaginalis $\mathrm{sp}$. nov. is proposed.

Cultures. Details of the strains and their sources are given in Table 1.

DNA base composition and DNA-DNA hybridization. Cells for deoxyribonucleic acid (DNA) isolation were grown in MRS broth (CM361; Oxoid Ltd.) at 25 or $37^{\circ} \mathrm{C}$ to the late exponential phase, harvested by centrifugation, and washed with Tris-EDTA buffer ( $10 \mathrm{mM}$ Tris, $1 \mathrm{mM}$ EDTA [pH 8.0]). DNA was prepared by a modification of the method of Garvie (2). DNA base composition was estimated from the midpoint of the DNA melting profile $\left(T_{m}\right)$ in $0.1 \times \mathrm{SSC}(1 \times$ $\mathrm{SSC}$ is $0.15 \mathrm{M} \mathrm{NaCl}$ plus $0.015 \mathrm{M}$ sodium citrate [pH 7.0]), with DNA from Escherichia coli $\mathrm{K}-12$ as a standard. Preliminary DNA hybridizations were done on Zeta-Probe nylon membranes (Bio-Rad Laboratories Ltd.) with a slot-blot apparatus (Bio-Rad) and probe DNA labeled with biotinUTP by nick translation (Bethesda Research Laboratories Ltd.). Hybridizations were carried out under semistringent conditions $\left(T_{m}-20^{\circ} \mathrm{C}\right)$. Quantitative data were generated with radiolabeled DNA and membrane filter hybridizations done under optimum conditions $\left(T_{m}-25^{\circ} \mathrm{C}\right)(2)$.

Fatty acid analyses. Dried biomass $(30 \mathrm{mg}$ ) of cells grown in yeast-glucose-phosphate broth (3) was degraded by overnight methanolysis at $55^{\circ} \mathrm{C}$. Long-chain fatty acid methyl esters were purified and analyzed by capillary gas chromatography as described previously (1). Capillary gas chromatography-mass spectrometry of picolinyl esters of fatty acids was done as described by Wait and Hudson (7).

Biochemical tests. The API $50 \mathrm{CH}$ system was used in accordance with the instructions of the manufacturer (APIBioMerieux Ltd.).Tests were incubated at 30 and $37^{\circ} \mathrm{C}$ and read after 24 and $48 \mathrm{~h}$ and after 7 days. The products of

\footnotetext{
* Corresponding author.
}

glucose fermentation were determined by gas chromatography as described in the Anaerobe Laboratory Manual (4). The isomer of lactic acid produced by the strains in MRS broth was determined with a commercial test kit (Boehringer Mannheim Biochemicals).

The guanine-plus-cytosine compositions of the five strains of $L$. vaginalis varied between 38 and $41 \mathrm{~mol} \%$. The results of preliminary slot blot hybridizations indicated that they were homogeneous and distinct from reference strains of $L$. fermentum and $L$. reuteri. The results of quantitative DNADNA hybridizations are shown in Table 1 . The strains of $L$. vaginalis formed a single homology group that was 85 to $90 \%$ related to the type strain $L$. vaginalis NCTC 12197 . Strains of $L$. oris and $L$. reuteri were most closely related to $L$. vaginalis NCTC $12197^{\mathrm{T}}$ at 26 to $29 \%$ and 24 to $35 \%$ homology, respectively. L. fermentum and the remaining reference strains were only distantly related $(\leq 18 \%)$ to $L$. vaginalis. If $70 \%$ homology under optimum conditions is used as a guideline for species differentiation (5), the DNA data clearly demonstrate that $L$. vaginalis is a distinct taxon worthy of separate specific status. Biochemical features for the differentiation of $L$. vaginalis from $L$. fermentum, $L$. oris, and $L$. reuteri are given in Table 2 . A full description of $L$. vaginalis is given below.

Description of Lactobacillus vaginalis sp. nov. Lactobacillus vaginalis (vaginalis. L. gen. n. vaginalis, of the vagina). Cells are rod shaped, are of variable size $(0.5$ to 0.8 by 1.5 to $25 \mu \mathrm{m}$ ), and occur singly or in pairs or short chains. Colonies are white to grey, small to large ( 1 to $5 \mathrm{~mm}$ ), and semirough, often with raised areas. Cells are gram positive. Nonmotile. Grows at 30 and $45^{\circ} \mathrm{C}$ but not at 22 or $48^{\circ} \mathrm{C}$. Facultatively anaerobic. Catalase negative. Heterofermentative, producing D- and L-lactic acid. Acid is produced from D-galactose, D-glucose, lactose, D-levulose, D-mannose, maltose, melibiose, D-raffinose, and sucrose. Most strains produce acid from ribose. Acid is not produced from $N$-acetylglucosamine, adonitol, D-arabinose, L-arabitol, arbutin, cellobiose, dulcitol, erythritol, D- and L-fucose, glycerol, glycogen, inositol, inulin, 2- and 5-ketogluconate, D-lyxose, mannitol, melezitose, $\alpha$-methyl-D-mannoside, rhamnose, sorbitol, Lsorbose, starch, D-tagatose, trehalose, D-turanose, xylitol, and D- and L-xylose. Esculin is hydrolyzed slowly by most strains. Produces straight-chain and monounsaturated longchain fatty acids of the vaccenic series and cis-11,12-methylene-octadecenoic (lactobacillic) acid. The guanine-pluscytosine content of the DNA ranges from 38 to $41 \mathrm{~mol} \%$ $\left(T_{m}\right)$. Isolated from the vagina of patients suffering from trichomoniasis. 
TABLE 1. Strain histories, DNA base compositions, and DNA homologies of $L$. vaginalis and other lactobacilli

\begin{tabular}{|c|c|c|c|}
\hline Species and strain & $\begin{array}{l}\text { Source or other } \\
\text { description }\end{array}$ & 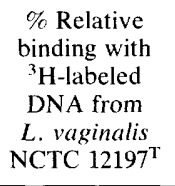 & $\begin{array}{c}\text { Guanine- } \\
\text { plus- } \\
\text { cytosine } \\
\text { content } \\
\text { (mol\%) }\end{array}$ \\
\hline \multicolumn{4}{|l|}{ L. vaginalis } \\
\hline NCTC $12197^{T}$ & Human vagina & 100 & 39 \\
\hline 4 & Human vagina & 86 & 38 \\
\hline 6 & Human vagina & 85 & 38 \\
\hline 8 & Human vagina & 90 & 39 \\
\hline 10 & Human vagina & 99 & 41 \\
\hline $\begin{array}{l}\text { L. acidophilus } \\
\text { NCDO } 1748^{\mathrm{T}}\end{array}$ & $\begin{array}{l}\text { ATCC } 4356^{\mathrm{T}}, \text { DSM } \\
20079^{\mathrm{T}}\end{array}$ & 16 & 34 \\
\hline $\begin{array}{l}\text { L. agilis NCDO } \\
2744^{\mathrm{T}}\end{array}$ & $\begin{array}{l}\text { DSM } 20509^{\mathrm{T}}, \text { NCIB } \\
11716^{\mathrm{T}} ; \text { municipal } \\
\text { sewage }\end{array}$ & 13 & 41 \\
\hline \multicolumn{4}{|l|}{ L. brevis } \\
\hline NCDO 391 & $\begin{array}{l}\text { ATCC 4356, DSM } \\
20079\end{array}$ & 16 & 34 \\
\hline NCDO 476 & & 12 & 44 \\
\hline $\begin{array}{l}\text { L. buchneri } \\
\text { NCDO } 110^{\mathrm{T}}\end{array}$ & $\begin{array}{l}\text { ATCC } 4005^{\mathrm{T}}, \text { DSM } \\
20057^{\mathrm{T}} \text {; tomato } \\
\text { pulp }\end{array}$ & 11 & 45 \\
\hline $\begin{array}{l}\text { L. casei } \mathrm{NCDO} \\
161^{\mathrm{T}}\end{array}$ & $\begin{array}{l}\text { ATCC } 393^{\mathrm{T}}, \text { DSM } \\
20011^{\mathrm{T}}\end{array}$ & 10 & $\mathrm{ND}^{a}$ \\
\hline $\begin{array}{l}\text { L. collinoides } \\
\text { NCDO } 2746^{T}\end{array}$ & $\begin{array}{l}\text { ATCC } 27612^{\mathrm{T}}, \text { DSM } \\
20515^{\mathrm{T}}, \mathrm{NCIB} \\
10925^{\mathrm{T}}\end{array}$ & 8 & 47 \\
\hline $\begin{array}{l}\text { L. confusus } \\
1635-85\end{array}$ & Human & 12 & ND \\
\hline $\begin{array}{l}\text { L. curvatus NCDO } \\
2739^{\mathrm{T}}\end{array}$ & DSM 20019 ; milk & 13 & ND \\
\hline $\begin{array}{l}\text { L. falsus NCDO } \\
151\end{array}$ & Originally $L$. casei & 8 & ND \\
\hline $\begin{array}{l}\text { L. farciminis } \\
\text { NCDO } 2330^{\mathrm{T}}\end{array}$ & $\begin{array}{l}\text { ATCC } 29644^{\mathrm{T}}, \text { DSM } \\
20184^{\mathrm{T}} \text {; sausage }\end{array}$ & 11 & 36 \\
\hline $\begin{array}{l}\text { L. fermentum } \\
\text { NCDO } 217\end{array}$ & $\begin{array}{l}\text { M. E. Sharpe } \\
\text { (strain F3) }\end{array}$ & 17 & ND \\
\hline NCDO 223 & $\begin{array}{l}\text { M. E. Sharpe } \\
\text { (strain F4) }\end{array}$ & 17 & ND \\
\hline $\begin{array}{l}\text { L. gasseri NCDO } \\
2233\end{array}$ & $\begin{array}{l}\text { ATCC } 33323, \text { DSM } \\
20243, \text { NCIB } \\
11718\end{array}$ & 10 & 34 \\
\hline L. helveticus J11 & M. E. Sharpe & 13 & ND \\
\hline $\begin{array}{l}\text { L. hilgardii NCDO } \\
264^{T}\end{array}$ & $\begin{array}{l}\text { ATCC } 8290^{\mathrm{T}}, \text { DSM } \\
20176^{\mathrm{T}} ; \text { wine }\end{array}$ & 11 & 40 \\
\hline $\begin{array}{l}\text { L. intestinalis } \\
\text { NCDO } 2176\end{array}$ & HE1 & 10 & 37 \\
\hline $\begin{array}{l}\text { L. kefir NCDO } \\
2132\end{array}$ & Kefir & 12 & 41 \\
\hline $\begin{array}{l}\text { L. malefermentans } \\
\text { NCDO } 2344\end{array}$ & $\begin{array}{l}\text { DSM 20177, JCM } \\
\quad 1189\end{array}$ & 13 & 41 \\
\hline L. minor NCDO & O. Kandler & 11 & 38 \\
\hline \multicolumn{4}{|l|}{ L. oris } \\
\hline NCDO $2160^{\mathrm{T}}$ & $\begin{array}{l}\text { NCIB } 8831^{\mathrm{T}} \text {; human } \\
\text { saliva }\end{array}$ & 29 & 49 \\
\hline NCDO 2161 & Human saliva & 26 & 49 \\
\hline NCDO 2164 & Human saliva & 26 & 50 \\
\hline $\begin{array}{l}\text { L. parabuchneri } \\
\text { NCDO } 2748\end{array}$ & Human saliva & 12 & 44 \\
\hline $\begin{array}{l}\text { L. pentosus } \\
\text { NCDO } 363^{\mathrm{T}}\end{array}$ & Corn silage & 10 & 41 \\
\hline $\begin{array}{l}\text { L. plantarum } \\
\text { NCDO } 1752^{\mathrm{T}}\end{array}$ & $\begin{array}{l}\text { HuATCC } 14917^{\mathrm{T}} \\
\text { DSM } 20174^{\mathrm{T}} \text {; } \\
\text { pickled cabbage }\end{array}$ & 11 & 44 \\
\hline
\end{tabular}

TABLE 1.-Continued

\begin{tabular}{|c|c|c|c|}
\hline $\begin{array}{l}\text { Species and } \\
\text { strain }\end{array}$ & $\begin{array}{l}\text { Source or other } \\
\text { description }\end{array}$ & $\begin{array}{c}\% \text { Relative } \\
\text { binding with } \\
{ }^{3} \mathrm{H} \text {-labeled } \\
\text { DNA from } \\
\text { L. vaginalis } \\
\text { NCTC } 12197^{\mathrm{T}}\end{array}$ & $\begin{array}{c}\text { Guanine } \\
\text { plus- } \\
\text { cytosine } \\
\text { content } \\
\text { (mol\%) }\end{array}$ \\
\hline $\begin{array}{l}\text { L. reuteri } \\
\text { NCDO } 2589^{\mathrm{T}}\end{array}$ & $\begin{array}{l}\text { ATCC } 23272^{\mathrm{T}} \text {, DSM } \\
20016^{\mathrm{T}} \text {; adult human } \\
\text { intestine }\end{array}$ & & \\
\hline NCDO 1089 & M. E. Sharpe & 33 & 38 \\
\hline NCDO 219 & M. E. Sharpe & 24 & 38 \\
\hline NCDO 230 & M. E. Sharpe & 27 & 39 \\
\hline NCDO 2655 & Rat & 28 & 40 \\
\hline $\begin{array}{l}\text { L. rhamnosus } \\
\text { NCDO } 86\end{array}$ & $\begin{array}{l}\text { ATCC } 11977, \text { NCIB } \\
76, \text { NCTC } 76\end{array}$ & 9 & ND \\
\hline $\begin{array}{l}\text { L. viridescens } \\
\text { NCDO } 1655^{\mathrm{T}}\end{array}$ & $\begin{array}{l}\text { ATCC } 12706^{\mathrm{T}}, \text { DSM } \\
20410^{\mathrm{T}}\end{array}$ & 15 & 43 \\
\hline NCDO 403 & C. F. Niven & 14 & 43 \\
\hline
\end{tabular}

${ }^{a} \mathrm{ND}$, Not determined.

TABLE 2. Differential features of $L$. vaginalis, $L$. fermentum, L. oris, and L. reuteri ${ }^{a}$

\begin{tabular}{|c|c|c|c|c|}
\hline \multirow{2}{*}{$\begin{array}{l}\text { Acid produced } \\
\text { from }^{b}:\end{array}$} & \multicolumn{4}{|c|}{ No. of the following strains positive ${ }^{c}$ : } \\
\hline & $\begin{array}{l}\text { L. vaginalis } \\
(n=6)\end{array}$ & $\begin{array}{l}\text { L. fermentum } \\
(n=13)\end{array}$ & $\begin{array}{l}\text { L. oris } \\
(n=5)\end{array}$ & $\begin{array}{l}\text { L. reuteri } \\
(n=8)\end{array}$ \\
\hline Amygdalin & - & - & + & - \\
\hline L-Arabinose & - & 3 & + & 7 \\
\hline D-Arabitol & - & - & + & - \\
\hline Esculin & $4^{\mathrm{D}}$ & - & 3 & 1 \\
\hline D-Fructose & + & 11 & + & - \\
\hline D-Furanose & - & - & 3 & - \\
\hline Gentiobiose & - & - & + & - \\
\hline Gluconate & - & $t^{D}$ & 4 & 5 \\
\hline D-Mannose & + & 6 & 1 & - \\
\hline$\alpha$-Methylglucoside & - & 4 & 4 & 2 \\
\hline$\alpha$-Methylxyloside & - & 1 & $3^{\mathrm{D}}$ & - \\
\hline Salicin & - & - & 3 & - \\
\hline Sucrose & + & 10 & + & + \\
\hline
\end{tabular}

"The guanine-plus-cytosine contents for $L$. vaginalis, $L$. fermentum, $L$. oris, and $L$. reuteri were 38 to 41,42 to 53,49 to 51 , and 37 to $41 \mathrm{~mol} \%$, respectively.

${ }^{b}$ All L. vaginalis strains produced acid from ribose (after 7 days), Dgalactose, D-glucose, lactose, maltose, melibiose, D-raffinose, and saccharose; all $L$. vaginalis strains failed to produce acid from $N$-acetylglucosamine, adonitol, D-arabinose, L-arabitol, arbutin, cellobiose, dulcitol, erythritol, Dand L-fucose, glycerol, glycogen, inositol, inulin, 2- and 5-ketogluconate, D-lyxose, mannitol, melezitose, $\alpha$-methyl-D-mannoside, rhamnose, sorbitol, L-sorbose, starch, D-tagatose, trehalose, D-turanose, xylitol, and D- and L-xylose.

'+, All strains positive; - , all strains negative; $\mathrm{D}$, delayed reaction.

The type strain is NCTC 12197 . In most respects the description of the type strain resembles the species description. The type strain does not produce acid from ribose and does not hydrolyze esculin.

We thank R. Wait for mass spectrometry of picolinyl esters.

\section{LITERATURE CITED}

1. Embley, T. M., R. Waite, G. Dobson, and M. Goodfellow. 1987. Fatty acid composition in the classification of Saccharopolyspora hirsuta. FEMS Microbiol. Lett. 41:131-135.

2. Garvie, E. I. 1976. Hybridisation between the deoxyribonucleic acids of some heterofermentative lactic acid bacteria. Int. J. 
Syst. Bacteriol. 26:116-122.

3. Garvie, E. I. 1978. Streptococcus raffinolactis Orla-Jensen and Hansen, a group $\mathrm{N}$ streptococcus found in raw milk. Int. J. Syst. Bacteriol. 28:190-193.

4. Holdemann, L. V., E. P. Cato, and W. E. C. Moore (ed.). 1977. Anaerobe laboratory manual, 4th ed. Virginia Polytechnic Institute and State University, Blacksburg.

5. Johnson, J. L. 1986. Nucleic acids in bacterial classification, p. 972-975. In P. H. A. Sneath, N. S. Mair, M. E. Sharpe, and J. G.
Holt (ed.), Bergey's manual of systematic bacteriology, vol. 2 . The Williams \& Wilkins Co., Baltimore.

6. Stewart, C. S., S. H. Duncan, and H. J. Flint. 1988. Characteristics of isolates of Lactobacillus fermentum from the rumen of sheep. Lett. Appl. Microbiol. 6:133-135.

7. Wait, R., and M. J. Hudson. 1985. The use of picolinyl esters for the characterisation of microbial lipids: application to the unsaturated and cyclopropane fatty acids of Campylobacter species. Lett. Appl. Bacteriol. 1:95-99. 